\title{
Research on the Dynamic Characteristics of Relaxed Static Stability Control Aircraft
}

\author{
Zhang Jicheng, Wang Duo, Luo Kai, Yang Yueming \\ Aviation University of Air Force, Changchun, China 130022 \\ 66224909@qq.coml,
}

Keywords: relaxed static stabillity control; long period; short period.

\begin{abstract}
In this paper we analysis aircraft dynamic characteristic on relaxed static stabillity control from the concept of active control technology point of view, and calculate the dynamic characteristic of longitudinal. Through calculation, further reseaerch on the influencing of relaxed static stability on flight performance are carried out, Providing a certain reference for pilot to master the characteristics on relaxed static stabillity control in order to complete flight missions.
\end{abstract}

\section{Introduction}

After the 1980s, fighter agility referred to an important position. Simply, it is the so-called agile mobility and maneuverability combination. It includes the acceleration and deceleration rate of change, the angular velocity change rate, roll angular acceleration. How to make the aircraft to achieve high agility, must find a way from aircraft design, if the aircraft is still in accordance with the conventional design, it is difficult to make the aircraft with high agility, resulting in a relaxed static stability. Relaxed static stability as an active control technology in aircraft design refers to abandon traditional static stability requirement, designed to allow the aircraft to less stable or neutral static stability, even static instability, and thus aircraft stability and handling quality problems caused by the aid of the automatic control system to be addressed.

Relaxed static stability, including the relaxation of longitudinal static stability and relax Fangxiang Jing stability categories, similar to its basic principles, this article discusses only relaxed longitudinal static stability of the situation.

\section{Relaxed static stability}

The stability of aircraft including static stability and dynamic stability. Static stability has been defined as the initial tendency that the airplane displays after being disturbed from its trimmed condition. Occasionally, the initial tendency is different or opposite from the overall tendency, so distinction must be made between the two. Dynamic stability is the overall tendency that the airplane displays after its equilibrium is disturbed.

Usually used $m_{z}^{c_{y}}=\bar{X}_{G}-\bar{X}_{F}$ as the basis to determine whether the aircraft have longitudinal static stability; if $m_{z}^{c_{y}}<0$, the plane is longitudinal static stability; else if $m_{z}^{c_{y}}=0$, the aircraft is neutral static stability; else $m_{z}^{c_{y}}>0$, the aircraft is unstable.

Conventional aircraft generally must have sufficient static stability, that is to say $m_{z}^{c_{y}}<0$, Which require the focus of the aircraft prior to the center of gravity. During the transonic phase, the focus position of the aircraft the rapid shift aft with the flight Mach number increase , the aircraft will have too much AOA static stability $\left|m_{z}^{c_{y}}\right|$ for supersonic flight. This will bring a range of issues. First, when flying at supersonic speeds, the trim deflected angle of horizontal tail (negative) will increase, in order to make the deflected angle of horizontal tail decreasing used for maneuver, moreover, with the elevator angle for unit overload increasing, the maneuver of the aircraft will be reduced; Secondly, the trim deflected angle and the loads of the horizontal tail are large, that will 
inevitably lead to the increase in weight of aircraft structure, so that the aircraft flight performance will be worse; Third, if the trim deflected angle of the horizontal tail increase, the negative lift of the horizontal tail will increse, leading to the loading of the wing increases, making the real aerodynamic characteristics of aircraft deterioration. These problems to the high performance fighter of large sweep and low aspect ratio are more serious. Relaxed static stability is designed in order to solve these problems, puts forward to improve of aircraft performance.

\section{Examples and analyses}

Longitudinal static stability coefficient for a certain type of aircraft:

$\frac{-M_{z}^{\alpha}}{I_{z}}=\bar{X}_{G}-\bar{X}_{G} \frac{b_{A}}{I_{z}} Y^{\alpha}=8.574-59.49952 \Delta \bar{X}_{G}$

Indicated that, when $\Delta \bar{X}_{G}=0.144102, \frac{-M_{z}^{\alpha}}{I_{z}}=0$, that is $M_{z}^{\alpha}=0$ 。

The characteristic determinant is:

$\Delta=\left|\begin{array}{ccc}s+0.0166 & -0.008 & 0.0369 \\ 0.105 & s+0.585 & -s \\ -0.02 & -(0.248 s+8.574) & s(s+0.627)\end{array}\right|+59.49952 \Delta \bar{X}_{G}\left|\begin{array}{cc}s+0.0166 & 0.0369 \\ 0.105 & -s\end{array}\right|=0$

The upper formula was launched:

$\Delta=\left(s^{4}+1.4766 s^{3}+8.73787 s^{2}+0.1465275 s+0.033652\right)-59.49952 \Delta \bar{X}_{G}\left(s^{2}+0.0166 s+0.0038745\right)=0$ open-loop transfer function is

$\frac{Z(s)}{N(s)}=\frac{-59.49952 \Delta \bar{X}_{G}\left(s^{2}+0.0166 s+0.0038745\right)}{\left(s^{2}+0.0161744 s+0.0038634\right)\left(s^{2}+1.46043 s+8.710385\right)}$
$\frac{Z(s)}{N(s)}=\frac{-59.49952 \Delta \bar{X}_{G}\left(s^{2}+0.0166 s+0.0038745\right)}{\left(s^{2}+0.0161744 s+0.0038634\right)\left(s^{2}+1.46043 s+8.710385\right)}:-0.0083 \pm 0.0617 \mathrm{i}$

Zero point: $-0.0083 \pm 0.0617 \mathrm{i}$

Long period mode pole: $-0.0080872 \pm 0.061628 \mathrm{i}$

Short period mode pole: $-0.730215 \pm 2.859575 \mathrm{i}$

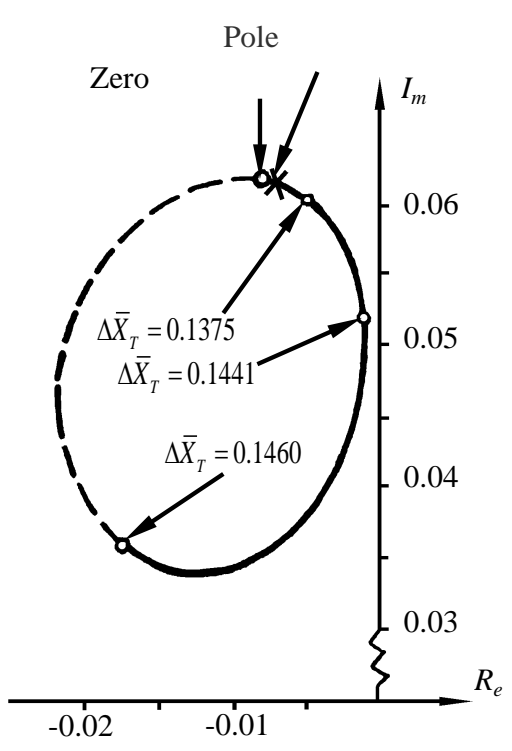

Fig.1 The root locus of long period mode

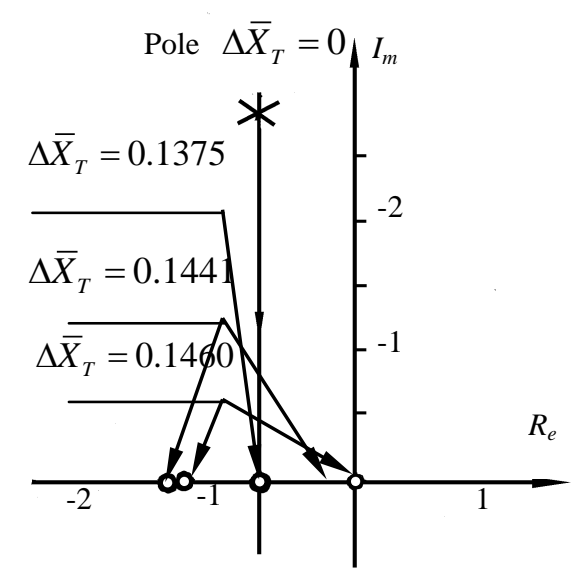

Fig.2 The root locus of short period mode 
Obviously, the zero point and long period mode pole close, can cancel each other out. The root locus of long period mode as shown in Figure 1. As can be seen from the figure, when the center of gravity shifts aft, the change of root locus only in a small region, namely the long period is always stable.

The root locus of short period mode as shown in Figure 2. As can be seen, when $\Delta \bar{X}_{G}=0.144102$ (i.e. $m_{z}^{\alpha}=0$ ), the short period mode is still stable, and then only when the center of gravity further shift, it would appear monotonous divergence, which indicates the stability of longitudinal disturbed motion is not only depends on $m_{z}^{\alpha}$.

If the center of gravity moves after the focus by $0.1 \mathrm{bA}$, it should be

$$
\begin{aligned}
\Delta \bar{X}_{T}= & 0.144102+0.1=0.244102 \text { So, } \\
\frac{-M_{z}^{\alpha}}{I_{z}} & =\bar{X}_{G}-\bar{X}_{G} \frac{b_{A}}{I_{z}} Y^{\alpha} \\
& =8.574-59.49952 \times 0.244102=-5.95
\end{aligned}
$$

In the formula (1), taking approximate short period characteristic determinant, and converting 8.574 into -5.95 , because of considering the gravity center displacement. Get

$\Delta=\left|\begin{array}{cc}s+0.585 & -s \\ 0.2482 s-5.95 & (s+0.627) s\end{array}\right|=0$

Expand, get

$s\left(s^{2}+1.46 s-5.583205\right)=0$

Solution to three real roots:

$\mathrm{S} 1=0, \mathrm{~S} 2=-3.203076, \mathrm{~S} 3=1.743076$

Analysis, $\mathrm{S} 1=0$, no practical significance; $\mathrm{S} 2=-3.203076$, is fast mode; $\mathrm{S} 3=1.743076$, is monotonic divergent modes. Therefore, moving the center of gravity after the focus position, the aircraft is statically unstable, and the dynamic stability is decreased or even dynamic instability.

\section{Conclusion}

Relaxed static stability requirements can improve the aircraft horizontal acceleration performance and maneuvers available overload.

Relaxed static stability can make $m_{z}^{c_{y}}\left(\right.$ or $m_{z}^{\alpha}$ ) negative value decrease, the aircraft trim drag reduce, enable the aircraft horizontal acceleration increase.

The F-16 aircraft center of gravity position moved from $25 \%$ bA to $38 \%$ bA, the required accelerate time is shortened 1.8s, when the Mach number of aircraft grown from 0.9 to 1.6.

The requirements for relaxed static stability, can reduce the aircraft's trim angle of attack, in certain circumstances of the maximum angle of attack, the available angle of attack in maneuver flight increase. Further, longitudinal static stability decreases, $m_{z}^{c_{y}}$ (or $m_{z}^{\alpha}$ ) negative value decreases, so the elevator angle for unit overload $\left|d \delta_{z} / d n_{y}\right|$ decreases, which will allow the maximum lift coefficient significantly increased when supersonic flying, rather than limited by the elevator angle,so that the available normal overload increases.

Available normal overload increases, will inevitably lead to the normal circling radius decreases, the angular velocity of circling increases, makeing the level of maneuverability better. Of course, the available angle of attack for maneuver flight increases, the available lift for maneuver flight increase, also bring significant benefits for vertical mobility.

\section{Acknowledgment}

This paper supported by National Natural Science Foundation China (Grant NO: 61172126/ F0 10410) 


\section{References}

[1]Lin Guohua editor aircraft flight performance and control Xi'an: Air Force Engineering Institute, 1997.1

[2]Chenting Nan editor application hydrodynamics Beijing: Aviation Industry Press, 2000.8

[3]Yang Yueming editor flight control principle Beijing: Blue Sky Press, 2004.11

[4]Gu Songfen editor aircraft design. Beijing: Beijing University of Aeronautics and Astronautics Press, 2001.9

[5]Taomei Zhen editor integrated design of modern aircraft structures Xi'an: Northwestern University Press, 2001.9

[6]Shi Following edited by the flight control system and enhance Beijing: National Defense Industry Press, 2003.1

[7] Yan Chao, Yu Jianjun, Li Junzhe. Research on CFD Scheme and Grid Effect in heat flux [J]. Aerodynamic Transaction of China, Vol.24, 2006.

[8]Chpoun A, Lengrand J C, Heffner K S. Numerical and Experimental Investigation of Rarefied Compression Corner Flow [R]. AIAA 92-2900, 1992.

[9] Boylan D E. Laminar Heat Transfer on Sharp and Blunt Ten-Degree Cones in Conical and Parallel Low Density Flow [C]. Arnold Engineering Development Center, Tullahorna, TN, AEDC-TR-73-106, Aug. 1973

[10]Lockerby D A, Reese J M, Emerson D R, Barber R W. Velocity Boundary Condition at Solid Walls in Rareedgas Calculations [J]. Physical Review E, 70(017303), 2004.

[11] Gokcen T, MacCormack R W. Nonequilibrium Effects for Hypersonic Transitional Flows Using Continuum Approach [R]. AIAA Paper 1989-0461, 1989.

[12] Lockerby D A, Reese J M, Gallis M A. Capturing the Knudsen Layer in Continuum-Fluid Models of Nonequilibrium Gas Flows [J]. AIAA Journal, 43(6), 2005.

[13] Liu Xuewei, Luo Kai. Numerical Simulation on Computational Model of Hypersonic Slip Flow [C]. ICSMMS 2014.

[14] Luo Kai, Pan Sha, Li Hua. Research on Computational Heat Flux Model in Hypersonic Slip Flow [C]. Dynamical Branch of Aerodynamic Academy in China, Chengdu, 2011.

[15] Lofthouse A J, Boyd I D. Hypersonic Flow over a Flat Plate: CFD Comparison with Experiment [R]. AIAA 2009-1315, 2009.

[16] Yan Chao, Yu Jianjun, Li Junzhe. Research on CFD Scheme and Grid Effects in Heat Flux Simulation [J]. Aerodynamic Transaction, Vol 24, 2006.

[17] Yan Chao, Yu Jianjun, Li Junzhe. Research on CFD Scheme and Grid Effect in heat flux [J]. Aerodynamic Transaction of China, Vol.24, 2006.

[18]Chpoun A, Lengrand J C, Heffner K S. Numerical and Experimental Investigation of Rarefied Compression Corner Flow [R]. AIAA 92-2900, 1992.

[19] Kim K H, Kim C. Accurate Efficient and Monotomic Numerical Methods for Multi-Dimensional Compressible Flows Part I: Spatial Discretization [J]. Journal of Computational Physics, 2005, 208.

[20]Yoon S, Jameson A. Lower-Upper Symmetric Gauss-Sediel Method for the Euler and Navier-Stokes Equations [J]. AIAA Journal, 1988, 26(9).

[21] Shihongchangetc. Wind tunnel data acquisition technology [M]. Beijing: National Defense Industry Press 2004.01

[22]Chen Maozhang. Foundations of Viscous Fluid Dynamics [M]. Beijing: High Education Press, 2002.

[23]Shihong Chang .etc Compile. wind tunnel data acquisition technology [M] .Beijing: National 
Defense Industry Press 2002.02

[24]Yunqilinzhu Wind tunnel experiment data error correction [M]. Beijing: National Defense Industry Press, 1996.1

[25]Liu zheng-chong. tunnel structure design[M] .Beijing: China Astronautic Publishing. 2005.01

[26] Wuronglin. Wind tunnel design principles [M]. Beijing: Beijing Institute of Aeronautics Press. 1985.10

[27] Li Hua, Tian Zhengyu. Numerical Simulation on the Effects of Hypersonic Slip Flow[C]. Near Space CFD Seminar, Beijing, 2010.

[28] Myong R S. Velocity Slip Effect in Low-Speed Microscale Gas [R]. AIAA Paper 2001-3076.

[29] Lockerby D A. Geometric and Constitutive Dependence of Maxwell's Velocity Slip Boundary Condition [R] ADA Report, 2005.

[30] Bailey. A Critical Review of the Drag Force on a Sphere in the Transition Flow Regime [R]. ADA Report, 2005.

[31] Lofthouse A J, Scalabrin L C, Boyd I D. Velocity Slip and Temperature Jump in Hypersonic Aerothermodynamics [R]. AIAA 2007-208, 2007.

[32] Chen Maozhang. Foundations of Viscous Fluid Dynamics [M]. Beijing: High Education Press, 2002.

[33] Kim K H, Kim C. Accurate Efficient and Monotomic Numerical Methods for Multi-Dimensional Compressible Flows Part I: Spatial Discretization [J]. Journal of Computational Physics, 2005, 208. 